\title{
Microleakage in Class II composite restorations with margins below the CEJ: In vitro evaluation of different restorative techniques
}

\author{
Claudio Poggio ${ }^{1}$, Marco Chiesa ${ }^{1}$, Andrea Scribante ${ }^{2}$, Jenia Mekler ${ }^{1}$, Marco Colombo ${ }^{1}$ \\ ${ }^{1}$ Department of Operative Dentistry, University of Pavia, Policlinico "San Matteo" \\ 2 Department of Orthodontics, University of Pavia, Policlinico "San Matteo"
}

Correspondence:

Department of Operative Dentistry

University of Pavia, Policlinico San Matteo

Piazzale Golgi 3, 27100 Pavia, Italy

claudio.poggio@unipv.it

Received: 13/03/2012

Accepted: 14/11/2012

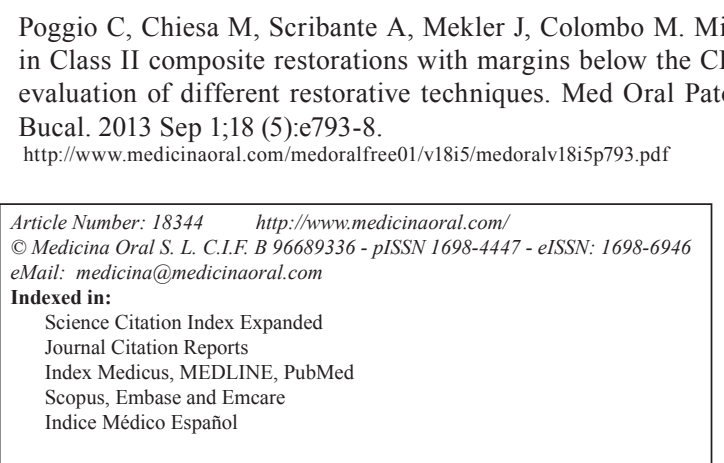

\begin{abstract}
Objectives: The purpose of this in vitro study was to evaluate the microleakage in "deep" Class II composite restorations with gingival cavosurface margin below the CEJ (cemento-enamel junction) and restored with different techniques.

Study Design: Fifty human teeth were used. In each tooth two standardized Class II slot cavities (on mesial and on distal surfaces) were prepared: the buccolingual extension of the cavities was $4 \mathrm{~mm}$; the gingival wall was located in dentin/cementum ( $2 \mathrm{~mm}$ beyond the CEJ). The prepared teeth were randomly assigned to 5 experimental groups (of 10 specimens and 20 cavities each) and restored. Group 1: Filtek TM Supreme XTE Flowable (3MESPE) + Universal Filtek Supreme XTE (3MESPE), Group 2: GrandioSO Heavy Flow (Voco) + GrandioSo (Voco), Group 3: SDR ${ }^{\mathrm{TM}}$ (Dentsply Caulk) + Esthet-X® HD (Dentsply Caulk), Group 4: SonicFill (Kerr), Group 5: Grandio (Voco). After thermocycling, the specimens were immersed in a $0.5 \%$ basic fuchsine dye solution and incubated at $37^{\circ} \mathrm{C}$ for 24 hours. The teeth were subsequently sectioned mesiodistally. All specimens were examined at $25 \times$ in a stereomicroscope and standardized digital images were obtained. Dye penetration was measured from gingival margins.

Results: The results demonstrated no significant leakage differences between Group 4 and Group 5, that both showed significantly higher frequency distribution of Score 0 . Group 2 and Group 3 showed a significant prevalence of Score 1, whereas Group 1 showed significantly higher frequency of Score 2.

Conclusions: None of the restorative techniques tested completely eliminated microleakage dye penetration in dentin margins; marginal adaptation in Class II composite restorations with gingival wall below the CEJ varied in both substrates and from different restorative techniques used.
\end{abstract}

Key words: Microleakage, Class II composite restorations, CEJ. 


\section{Introduction}

Direct Class II composite restorations can be placed at an acceptable standard if the cervical margin is in sound enamel; when the adhesive restorations are located below the CEJ (cemento-enamel junction) and cervical lesions have no enamel the quality of the marginal integrity is questionable (1). Below the CEJ the bond with dentin is weaker: the polymerization shrinkage can result in gap formation between composite resin and the cavity walls. Marginal gap formation contributes to microleakage permitting the passage of oral fluids and bacteria from the oral cavity and become a source of post-operative sensitivity, pulpal inflammation and recurrent caries (2-4). To reduce these effects have been suggested, as a better option to the conventional resin technique, the Class II open-sandwich restorations: glass-ionomer cement (GIC) or resin-modified glass-ionomer cement (RMGIC) is placed between the dentin cervical margins and occlusal composite restoration $(5,6)$. GICs and RMGICs have been shown to be less able to seal margins, can dissolve over time in the oral environment (7-9). Recently flowable resin composites (FRC), with lower filler content and far lower viscosity, have been recommended as liners at CEJ margins of the proximal box of Class II composite restorations to improving marginal integrity and to resulting less microleakage and post-operative sensitivity: $(4,10)$ a layer of flowable materials at the gingival floor (in cementum margins) of Class II composite restorations get better the marginal seal of a restoration and is an ideal choice for use in a open-sandwich technique (11-13). One approach to improving the marginal seal and reducing microleakage is to use a flowable composite resin under highly filled composite restorations: however several studies do not show improved performance $(10,14)$. The most recent attempt to reduce microleakage uses new resin monomers with novel chemistries (low polimerization shrinkage) to compensate shrikage stress. SDR ${ }^{\mathrm{TM}}$ (Dentsply Caulk) is designed to reduce microleakage by increasing flow with a unique chemistry that slows the rate of polimerization to reduce shrinkage stress This composite resin is used as a dentin replacement material and polymerized in 4- $\mathrm{mm}$ increments (15). SonicFill is a single-step composite system that doesn't require an additional capping layer. SonicFill System combines the advantages of a flowable composite with a universal composite. SonicFill System is comprised of a $\mathrm{KaVo}$ handpiece that enables sonic activation of a specially designed and conveniently delivered composite from Kerr. SonicFill's activation significantly reduces the composite's viscosity to rapidly fill the cavity. The purpose of this in vitro study was to evaluate the microleakage in "deep" Class II composite restorations with gingival cavosurface margin below the CEJ and restored with different techniques. The null hypothesis of the study was that there is no significant difference in microleakage of the different evaluated restorative techniques evaluated.

\section{Material and Methods}

-Specimen preparation

Fifty caries-free vital human teeth freshly extracted for periodontal or orthodontic reasons were used in this study. The teeth were cleaned with dental scalers, polished with pumice and stored in a $0.25 \%$ mixture of sodium azide in Ringer solution until the date of use. In each tooth two standardized Class II slot cavities (on mesial and on distal surfaces) were prepared with a round-nosed no.245 carbide bur (Dentsply/Caulk, Milford, DE, USA) at high-speed with air/water spray (16). The buccolingual extension of the cavities was $4 \mathrm{~mm}$; the gingival wall was located in dentin/cementum ( $2 \mathrm{~mm}$ below the cementumenamel junction/CEJ); the internal angles were rounded and cavosurface margins were finished with gingival margin trimmers (17). The prepared teeth were randomly assigned to 5 experimental groups (of 10 specimens and 20 cavities each) and were mounted in a jig featuring artificial training teeth that served as adjacent teeth. A contoured matrix band was placed around the teeth for restorative procedures. The same trained operator prepared all the cavities.

-Restorative procedure

Group 1. The cavities were etched with 37\% phosphoric acid for 30 seconds (Total Etch; Ivoclar Vivadent AG,Schaan,Liechtenstein) and bonded with Adper Scotchbond 1 XT (3M ESPE, St.Paul, MN, USA). A layer (approximately $1 \mathrm{~mm}$ in thickness) of a flowable material (Filtek TM Supreme XTE Flowable/3MESPE, St.Paul, MN, USA) was placed (by a periodontal probe) to cover the entire gingival floor of the cavity. The cavities were then restored with a "nanofilled" composite (Universal Filtek Supreme XTE/3MESPE, St.Paul, MN, USA), using a horizontal incremental technique with 3 horizontals increments ( $2 \mathrm{~mm}$ thick) from the cervical to the occlusal surface.

Group 2. The cavities were etched with 37\% phosphoric acid for 30 seconds (Total Etch; Ivoclar Vivadent AG,Schaan,Liechtenstein) and bonded with Adper Scotchbond 1 XT (3M ESPE,St.Paul,MN,USA). A layer (approximately $1 \mathrm{~mm}$ in thickness) of a flowable material (GrandioSO Heavy Flow/Voco GmbH, Cuxhaven, Germany) was placed (by a periodontal probe) to cover the entire gingival floor of the cavity. The cavities were then restored with a "nanoybrid" composite (GrandioSo/ Voco GmbH, Cuxhaven, Germany), using a horizontal incremental technique with 3 horizontals increments ( $2 \mathrm{~mm}$ thick) from the cervical to the occlusal surface. Group 3. The cavities were etched with 37\% phosphoric acid for 30 seconds (Total Etch; Ivoclar Vivadent AG,Schaan,Liechtenstein) and bonded with Adper Scotchbond 1 XT (3M ESPE,St.Paul,MN,USA). The flowable material (SDR ${ }^{\mathrm{TM}} /$ Dentsply Caulk,Mildford,DE,USA) was placed in a $4 \mathrm{~mm}$ bulk increments and light cured for 20 seconds. An occlusal layer of Esthet-X® HD 
(Dentsply Caulk, Mildford, DE, USA) composite was added on top to build the final anatomy of the teeth and to complete the restoration.

Group 4. The cavities were etched with 37\% phosphoric acid for 30 seconds (Total Etch; Ivoclar Vivadent AG,Schaan,Liechtenstein) and bonded with Adper Scotchbond 1 XT (3M ESPE, St.Paul, MN, USA). The flowable material (SonicFill/Kerr, West Collins, Orange, CA, USA) was placed with the SonicFill Handpiece (sonically activated delivery) in a $4 \mathrm{~mm}$ bulk increments and light cured for 20 seconds. SonicFill is a single-step composite system that doesn't require an additional capping layer.

Group 5. (control). The cavities were etched with 37\% phosphoric acid for 30 seconds (Total Etch; Ivoclar Vivadent $A G$, Schaan, Liechtenstein) and bonded with Adper Scotchbond 1 XT (3M ESPE, St.Paul, MN, USA). The cavities were totally restored with a "nanohybrid" composite (Grandio/Voco GmbH,Cuxhaven,Germany), using a horizontal incremental technique with 3 increments from the cervical to the occlusal surface (each increment being $2 \mathrm{~mm}$ ).

Each layer or increment was cured for 20 seconds from the occlusal surface with a LED curing light in softstart-polymerization mode (Celalux 2 High-Power LED curing-light, Voco $\mathrm{GmbH}$, Cuxhaven, Germany) for 20 seconds at a light intensity of $1000 \mathrm{~mW} / \mathrm{cm}^{2}$ according to manufacturers' instructions. Then the metallic matrix was removed and the restorations were light cured for 20 seconds from the buccal and lingual surfaces and the surface was finished and polished with finishing/polishing disks (Sof-Lex Pop-On, 3M ESPE, St. Paul, MN, USA) in decreasing granulation. All teeth were coated with two layers of nail varnish up to $1 \mathrm{~mm}$ from the restorations margins, while the apical part was sealed with wax. The restored teeth were then subjected to artificial aging by thermocycling. All specimens were immersed alternately in water baths at 5 and $60^{\circ} \mathrm{C}$ for $1500 \mathrm{cy}-$ cles, with at dwell time of 60 seconds in each bath and a transfer time of 15 seconds. After thermocycling, the specimens were immersed in a $0.5 \%$ basic fuchsine dye solution and incubated at $37^{\circ} \mathrm{C}$ for 24 hours. The teeth were subsequently rinsed for 10 minutes under running water to remove external dye, dried and sectioned mesiodistally through the centre of the restorations with a low-speed water-cooled diamond cutter.

-Microleakage analysis

All specimens were examined at $25 \mathrm{X}$ in a stereomicroscope (Inspective 4Geek, Serravalle, RSM) and standardized digital images were obtained. Two observers scored each section blindly; consensus was forced if disagreements occurred. Dye penetration was measured from gingival margins. An independent examiner did scoring; another trained examiner confirmed observations. The cervical marginal microleakage was recorded based on the following criteria (18): score $0=$ no dye penetration, score $1=$ dye penetration limited to enamel, score 2 = dye penetration beyond the dentinenamel junction but limited to $2 / 3$ rds of the cervical wall length, score $3=$ dye penetration beyond $2 / 3 \mathrm{rds}$ of the cervical wall length but not to the pulpal wall, score $4=$ dye penetration to the pulpal wall.

-Statistical analysis

The results of microleakage scores were subjected to statistical analysis using "Stata 7.0" computer software (Stata Corp., Station College, TX). As the data are on an ordinal scale, a Kruskal-Wallis test was used to assess differences among the different groups. Mann-Whitney U test was used as post hoc to investigate pairwise diffe-rences. Significance was predetermined for $\mathrm{P}<0.05$.

\section{Results}

Representative stereomicroscopic photograph of microleakage in Groups 1 to 5 are showed in figure 1. Microleakage scores for the dentin margins are presented in table 1 and illustrated in figure 2. The results demonstrated no significant leakage differences between Group 4 and Group 5, that both showed significantly higher frequency distribution of Score 0. Group 2 and Group 3 showed a significant prevalence of Score 1, whereas Group 1 showed significantly higher frequency of Score 2 (Fig. 2).

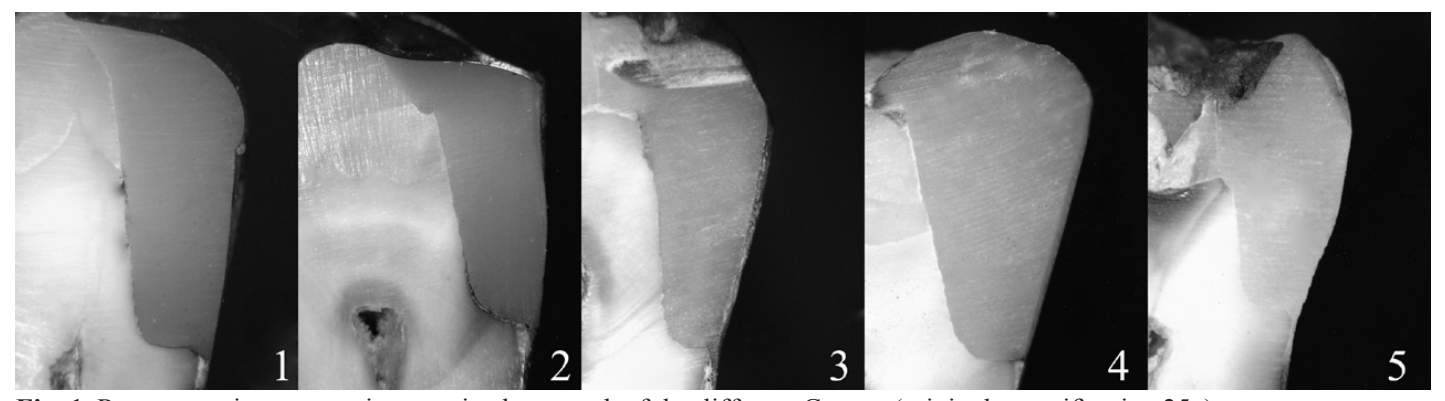

Fig. 1. Representative stereomicroscopic photograph of the different Groups (original magnification $25 \times$ ). 
Table 1. Frequency distributions of microleakage scores (percentages) on dentin margins among the different groups tested.

\begin{tabular}{|l|c|c|c|c|c|}
\hline & Score 0 & Score 1 & Score 2 & Score 3 & Score 4 \\
\hline Group 1 & $4(20 \%)$ & $3(15 \%)$ & $10(50 \%)$ & $1(5 \%)$ & $2(10 \%)$ \\
\hline Group 2 & $4(20 \%)$ & $9(45 \%)$ & $4(20 \%)$ & $1(5 \%)$ & $2(10 \%)$ \\
\hline Group 3 & $3(15 \%)$ & $10(50 \%)$ & $4(20 \%)$ & $2(10 \%)$ & $1(5 \%)$ \\
\hline Group 4 & $12(60 \%)$ & $5(25 \%)$ & $2(10 \%)$ & $1(5 \%)$ & $0(0 \%)$ \\
\hline Group 5 & $11(55 \%)$ & $7(35 \%)$ & $1(5 \%)$ & $1(5 \%)$ & $0(0 \%)$ \\
\hline
\end{tabular}

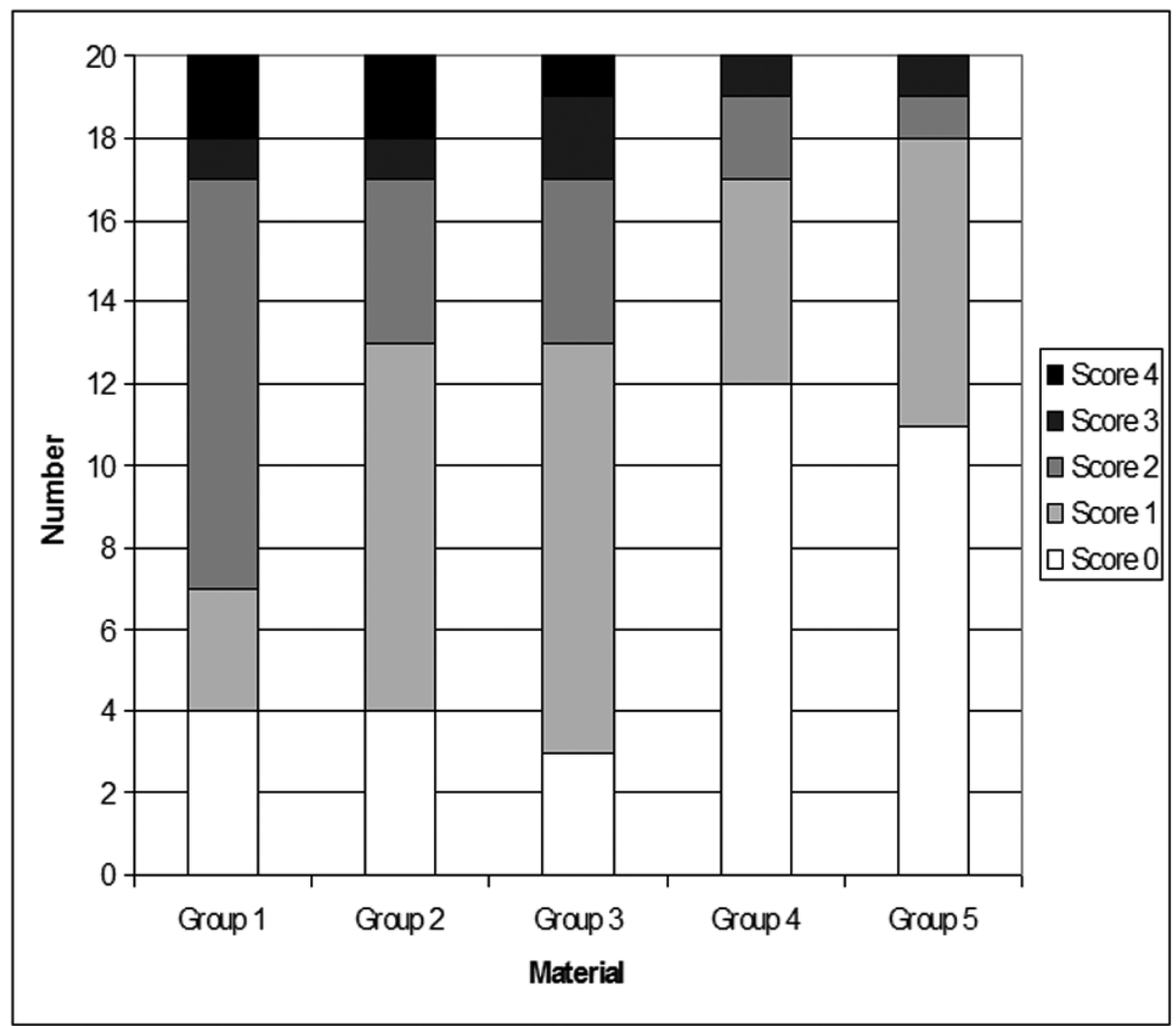

Fig. 2. Distribution of ARI scores of the different groups.

\section{Discussion}

The null-hypothesis of the study has been rejected. In the present investigation none of the adhesive systems tested completely eliminated microleakage in dentin margins of the cavity. This is in agreement with previous studies that evaluated microleakage of restoration at dentin interface $(12,19-21)$.

Significant prevalence of Score 0 (no dye penetration) was reported both for Groups 4 (SonicFill) and 5 (Grandio), thus indicating that both composites showed the lowest microleakage values when compared with other groups tested. Higher microleakage scores were recorded for Groups 2 (GrandioSO Heavy Flow+GrandioSO) and 3 (SDR+ Esthet-X HD), that both showed significant prevalence of Score 1 (dye penetration limited to enamel). The highest dye penetration values were reported for Group 1 (Filtek TM Supreme XTE Flowable+Universal Filtek Supreme XTE) that showed a significant prevalence of Score 2 (dye penetration beyond the dentinenamel junction but limited to $2 / 3$ rds of the cervical wall length). None of the Groups tested in the present investigation showed a significant prevalence of Score 3 and Score 4, thus indicating that the median of the scores reported was not correlated with a dye penetration beyond the dentin-enamel junction over $2 / 3$ rds of the cervical wall length or over pulpal wall (18).

Microleakage, due to microscopic openings between the margins of the composite restoration and the tooth, 
is considered a major cause of restoration failure (22). Dye penetration values obtained from in vitro studies are often higher than those obtained in vivo (23). In fact Microleakage tests have been widely employed to screen the seal efficiency of restorations. Such tests face the challenge of reproducing the oral dynamics in an in vitro assay. Their results tend to present high variability, probably, due to different test methods (24). Microleakage was chosen in this study because of its long-term report in literature. Furthermore, the test was designed taking into consideration the most frequent choices in test variables, as reported by Raskin, et al. (24) in a systematic literature review.

Microleakage can result in bacteria penetrating the toothrestoration space and into dentinal tubules, where secondary decay may occur and bacterial toxins will irritate the pulp. The oral environment (including occlusal forces and temperature variation) and several differences between the physical properties of teeth and restorative materials (including polymerization shrinkage, coefficient of thermal expansion, and modulus of elasticity) can contribute to microleakage (25). According to previous literature, if poor bond strength exists between the tooth and restorative material, a failure of adhesion may be caused by polymerization shrinkage, and microscopic gaps at the tooth/restoration interface can subsequently form $(26,27)$. Microleakage, either from small or microscopic openings between the margins of the composite restoration and tooth, was considered a major cause of restoration failure $(28,29)$. The majority of Class II cavities exhibit cavity margins with gingival wall below the CEJ in both dentine and/or cementum (30). Therefore, the cervical margins of restorations will be placed at dentine or cementum surfaces, which may lead to a weaker marginal seal than at the enamel surface (30). This in vitro study examined the microleakage in "deep" Class II composite restorations with gingival cavosurface margin below the CEJ and restored with different techniques. Within the limitations of this in vitro study, none of the restorative techniques tested completely eliminated microleakage dye penetration in dentin margins; marginal adaptation in Class II composite restorations with gingival wall below the CEJ varied in both substrates and from different restorative techniques used.

\section{References}

1. Yip KH, Poon BK, Chu FC, Poon EC, Kong FY, Smales RJ. Clinical evaluation of packable and conventional hybrid resin-based composites for posterior restorations in permanent teeth: Results at 12 months. J Am Dent Assoc. 2003;134:1581-9.

2. Attar N, Korkmaz Y. Effect of two light-emitting diode (LED) and one halogen curing light on the microleakage of Class $\mathrm{V}$ flowable composite restorations. J Contemp Dent Pract'. 2007;8:80-8.

3. Pashley DH. Clinical considerations of microleakage. J Endod. 1990;16:70-7.

4. Sadeghi M. The effect of fluid composite as gingival layer on microleakage of class II composite restorations. Dent. Res J. 2007;4:40-7.
5. Besnault C, Attal JP. Simulated oral environment and microleakage of class II resin-based composite and sandwich restorations. Am J Dent. 2003;16:186-90.

6. Loguercio AD, Reis A, Mazzocco KC, Dias AL, Busato AL, Singer JM, et al. Microleakage in class II composite resin restorations: total bonding and open sandwich technique. J Adhes Dent. 2002;4:137-44.

7. Holtan JR, Nystrom GP, Douglas WH, Phelps RA. Microleakage and marginal placement of a glass-ionomer liner. Quintessence Int. 1990;21:117-22.

8. Stockton LW, Tsang ST. Microleakage of Class II posterior restorations with gingival margins entirely within dentin. J Can Dent Assoc. 2007;73:255.

9. Welbury RR, Murray JJ. A clinical trial of the glass-ionomer cement-composite resin "sandwich" technique in Class II cavities in permanent premolar and molar teeth. Quintessence Int. 1990;21:507-12.

10. Neme AM, Maxson BB, Pink FE, Aksu MN. Microleakage of Class II packable resin composites lined with flowables: An in vitro study. Oper Dent. 2002;27:600-5.

11. Fabianelli A, Sgarra A, Goracci C, Cantoro A, Pollington S. Microleakage in class II restorations: open vs closed centripetal buildup technique. Oper Dent. 2010;35:308-3.

12. Sadeghi M. Influence of flowable materials on microleakage of nanofilled and hybrid Class II composite restorations with LED and QTH LCUs. Indian J Dent Res. 2009;20:159-63.

13. Ozgunaltay G., Görücü J. Fracture resistance of class II packable composite restorations with and without flowable liners. J Oral Rehabil. 2005;32:111-5.

14. Beznos C. Microleakage at the cervical margin of composite Class II cavities with different restorative techniques. Oper Dent. 2001;26:60-9.

15. Burgess J, Cakir D. Comparative properties of low-shrinkage composite resins. Compend Contin Educ Dent. 2010;31 Spec. No 2:10-5.

16. Rodrigues Junior SA, Pin LF, Machado G, Della Bona A, Demarco FF. Influence of different restorative techniques on marginal seal of class II composite restorations. J Appl Oral Sci. 2010;18:37-43.

17. Demarco FF, Ramos OL, Mota CS, Formolo E, Justino LM. Influence of different restorative techniques on microleakage in class II cavities with gingival wall in cementum. Oper Dent. 2001;26:253-9. 18. Chuang SF, Jin YT, Liu JK, Chang CH, Shieh DB. Influence of flowable composite lining thickness on Class II composite restorations. Oper Dent. 2004;29:301-8.

19. Osorio R, Toledano M, de Leonardi G, Tay F. Microleakage and interfacial morphology of self-etching adhesives in class $\mathrm{V}$ resin composite restorations. J Biomed Mater Res B Appl Biomater. 2003;66:399-409.

20. Koliniotou-Koumpia E, Dionysopoulos P, Koumpia E. In vivo evaluation of microleakage from composites with new dentine adhesives. J Oral Rehabil. 2004;31:1014-22.

21. Koubi S, Raskin A, Dejou J, About I, Tassery H, Camps J, et al. Effect of dual cure composite as dentin substitute on marginal integrity of class II open-sandwich restorations. Oper Dent. 2009;34:150-6.

22. Xie H, Zhang F, Wu Y, Chen C, Liu W. Dentine bond strength and microleakage of flowable composite, compomer and glass ionomer cement. Aust Dent J. 2008;53:25-31.

23. Malmström HS, Schlueter M, Roach T, Moss ME. Effect of thickness of flowable resins on marginal leakage in class II composite restorations. Oper Dent. 2002;27:373-80.

24. Raskin A, D'Horre W, Gonthier S, Degrange M, Déjou J. Reliability of in vitro microleakage tests: a literature review. J Adhes Dent. 2001;3:295-308.

25. Amaral CM, Hara AT, Pimenta LA, Rodrigues AL. Micro- leakage of hydrophilic adhesive systems in Class $\mathrm{V}$ composite restorations. Am J Dent. 2001;14:31-3.

26. Kubo S, Yokota H, Sata Y, Hayashi Y. Microleakage of self-etching primers after thermal and flexural load cycling. Am J Dent. 2001;14:163-9.

27. Retief DH, Mandras RS, Russell CM. Shear bond strength requi- 
red to prevent microleakage of the dentine /restoration interface. Am

J Dent. 1994;7:44-6.

28. Bergenholtz G, Cox CF, Loesche WJ, Syed SA. Bacterial leakage around dental restorations: its effect on the dental pulp. J Oral Pathol. 1982;11:439-50.

29. Jang KT, Chung DH, Shin D, Garcia-Godoy F. Effect of eccentric load cycling on microleakage of Class $\mathrm{V}$ flowable and packable composite resin restorations. Oper Dent. 2001;26:603-8.

30. Litonjua LA, Andreana S, Bush PJ, Tobias TS, Cohen RE. Noncarious cervical lesions and abfractions: a re-evaluation. J Am Dent Assoc. 2003;134:845-50. 